\title{
Relationship between selective visual attention and visual consciousness
}

\author{
Naotsugu Tsuchiya \\ Japan Science and Technology Agency, Japan \\ RIKEN Brain Science Institute, Japan \\ California Institute of Technology, USA
}

\section{Christof Koch \\ California Institute of Technology, USA}

The relationship between attention and consciousness is a close one, leading many scholars to conflate the two. However, recent research has slowly corroded a belief that selective attention and consciousness are so tightly entangled that they cannot be individually examined.

We distinguish between exogenous, saliency-driven, task-independent attention and top-down, endogenous and voluntary attention. In the first part we will summarize powerful computational models of saliency-driven attention that capture a large fraction of eye movements in normal subjects inspecting natural scenes.

In the second part, we will summarize psychophysical and neurophysiological evidence arguing that top-down attention and consciousness are distinct phenomena that need not occur together and that can be manipulated using distinct paradigms. Subjects can become conscious of an isolated object, or the gist of the scene in the near absence of top-down attention. Conversely, subjects can attend to perceptually invisible objects. We also cover the recent flurry of studies that utilized independent manipulation of attention and consciousness. These studies have shown paradoxical effects of attention, including examples where top-down attention and consciousness have opposing effects. These data provide clear evidence for distinctive influence of attention and consciousness on perception.

We argue that the dissociation is consistent with the following consideration of functions of attention and consciousness. The visual system is constantly bombarded with information, leading to a data deluge that cannot be processed in real time; on the order of one megabyte of raw information exits the retina every second. The prime goal of visual attention therefore is to select information to meet current behavioral goals. By definition this implies a relative decrease of processing resources for non-attended

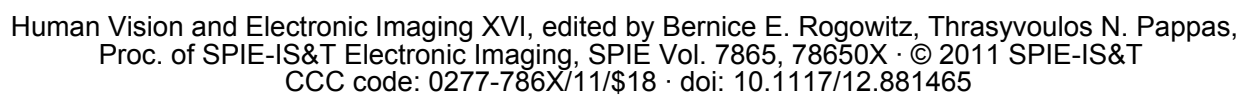


locations or features. Such attentional selection can be based on bottom-up, exogenous saliency-based factors or top-down, endogenous, goal-directed factors. Attention selects a portion of the input defined by a circumscribed region in space (spatial or focal attention), by a particular feature (feature-based attention), or by an object (object-based attention) for further processing.

Consciousness is surmised to have functions almost diametrically opposite to those of attention. It does not select information. Rather, proposed roles of consciousness include summarizing all relevant information pertaining to the current state of the organism and its environment and making this compact summary accessible to the planning stages of the brain, detecting anomalies and errors, decision making, language, inferring the internal state of other animals, setting long-term goals, making recursive models, and rational thought. This integrative aspect is emphasized by the Integrated Information Theory of consciousness. These functions also suggest that consciousness may be important at longer timescales than attention is, and may not be operating at the same level in the visual hierarchy. From this viewpoint, we can regard selective, focal attention as an analyzer and consciousness as a synthesizer.

Separating the effects of selective visual attention from those of visual consciousness is of paramount importance to untangle the neural substrates of consciousness from those for attention.

\section{Reference:}

Naotsugu Tsuchiya*, Jeroen J. A. van Boxtel*, Christof Koch "Attention and consciousness : on sufficiency and necessity" (2010) Frontiers of Consciousness Studies * equal contribution

http://www.frontiersin.org/consciousness_research/10.3389/fpsyg.2010.00217/abstract 